\title{
Primary Malignant Melanoma of the Vagina: Two Cases Reports
}

\author{
Achraf Hadiji, Tarak Damak, Ines Zemni, Riadh Chargui, Jamel Ben Hassouna, \\ Tarak Ben Dhiab, Monia Hechiche, Khaled Rahal. \\ Department of surgical oncology, Salah Azaiz Institute Bd 9 Avril Bab Saadoun 1009 Tunis Tunisia
}

\begin{abstract}
Aim: To report the experience in diagnosis, management and outcome of 2 cases of malignant melanoma of vagina.

Case Reports: the report concerns two postmenopausal patients with vaginal melanoma treated by radical surgery with bilateral inguinal pelvic lymphadenectomy. The first patient presented with a $4 \mathrm{~cm}$ pigmented brownish mass located in the posterior wall of the lower third of the vagina with a satellite nodule of $1 \mathrm{~cm}$ in the upper third of the vagina. The second patient had a $10 \mathrm{~cm}$ pigmented and ulcerated mass in the upper two third of posterior vaginal wall and an ulcer of $1.5 \mathrm{~cm}$ in the middle third of anterior vaginal wall. The first patient did not receive any adjuvant therapy after surgery and expired after one and half years because of local recurrence and distant metastasis. The second patient received radiotherapy after surgery and is alive for more than 2 years without any evidence of disease.

An adjuvant external radiotherapy at dose of 50Gy was delivered to the second patient and she was well 26 months later, with no sign of local or distant recurrence.

Conclusion: Though radical surgery is the treatment of choice for malignant melanoma of vagina adjuvant radiotherapy may improve survival by preventing recurrence.
\end{abstract}

\section{Introduction}

Vaginal melanoma is rare, accounting for less than $0.33 \%$ of melanomas and less than $3 \%$ of vaginal malignant tumors. In women, $1.6 \%$ of malignant melanomas are localized to the genital tract: the vulva, vagina and uterine cervix are involved in $70 \%, 21 \%$ and $19 \%$ of the cases, respectively [1-3].

It is an aggressive disease, with most patients diagnosed with advanced stages: $20 \%$ of patients present distant metastases at diagnosis and lymph node involvement is found in $50 \%$ of the cases $[4,5]$.

\section{Case 1}

A 70 year-old postmenopausal woman, was complaining of vaginal mass with pelvic pain since six months. Her medical history included subtotal hysterectomy for uterine leiomyoma. On physical examination, we found a $4 \mathrm{~cm}$ pigmented brownish mass located in the posterior wall of the lower third of the vagina with a second satellite nodule of $1 \mathrm{~cm}$ in the upper third of the vagina. No abnormalities were found on the examination of the uterine cervix and parametrium. Bilateral inguinal lymph nodes were not palpable. The chest X-ray and abdomino-pelvic tomography showed no sign of distant metastasis. An excision biopsy of the lesion taken under local anesthesia was reported as malignant nodular melanoma. Following the discussion of the case with multidisciplinary approach, it was decided to perform a total colpectomy with bilateral inguinal and pelvic lymphadenectomy. The patient was operated by abdomino-perineal approach. In the exploration of the abdomen, the abdominal organs were found normal and there was no grossly palpable lymph node in the pelvic and para aortic area.

The post-operative course was uneventful and the patient was discharged at $9^{\text {th }}$ days following surgery. The final pathology report concluded to the diagnosis of lentigous-type malignant melanoma with Breslow depth invasion of $0.6 \mathrm{~cm}$. On gross examination, we found a $1.2 \times 0.8 \mathrm{~cm}$ blackish pigmented nodule surrounded by pigmented plane area. Microscopically, the tumoral proliferation included rounded or ovoid cell with abundant cytoplasm containing melanic pigments. The nucleus was atypical with prominent nucleolus. High percentage of typical and atypical mitotic figures ( 9 per $10 \mathrm{hpf}$ ) was seen. The surgical margins of the specimen were negative for tumor cells. Only one lymph node was found to be involved in the right inguinal area of 17 removed lymph nodes. The sixteen removed pelvic lymph nodes were also tumor-free. The tumor staged T1N1M0 according to the American joint committee.

The patient presented a local recurrence, nine months after surgery, with $10 \mathrm{~mm}$ nodule located to the urethral opening, a multiple sub mucosal nodules involving the right labium majus and minus associated to a right inguinal lymph node recurrence. After discussion with the patient, a total vulvectomy with inguinal lymph node clearance was performed including the nodule located to the urethral opening. Six months later, the patient was diagnosed with multiple pulmonary and bone metastases. She received palliative radiotherapy to the vertebral column and she died before the beginning of the chemotherapy one month later. 


\section{Case 2}

A 75 year-old woman, presented at our institute with scanty spontaneous postmenopausal metrorrhagia lasting since 10 months. Her medical history included a hypertension, a facial paralysis with Claude Bernard Horner syndrome complicating the resection of cervical benign schwannoma. On the gynecological examination, a $10 \mathrm{~cm}$ pigmented and ulcerated mass was found in the upper two third of the posterior wall of the vagina associated to $1.5 \mathrm{~cm}$ ulceration in the anterior wall of the middle third. The uterine cervix, the uterus, the parametrium showed no abnormalities. No palpable lymph node was found on physical examination.

The biopsy concluded to the diagnosis of Superficial Spread Melanoma (SSM). The immunohistochemistry using monoclonal antibodies confirmed the diagnosis showing a positivity of the tumor cells for S100 protein and HMB-45 antibody and negativity for cytokeratin and epithelial membrane antigen.

The CT scan of the thorax, abdomen and pelvis, the rectoscopy, cystcopy showed no evidence of local or distant metastasis with any lymphadenomegaly.

After multidisciplinary discussion, the patient underwent a total hysterectomy with bilateral salpingo oophorectomy and a total colpectomy by abdomino-perineal approach. A bilateral iliac lymph node clearance was performed. The post-operative course was uneventful and the patient was discharged on the $8^{\text {th }}$ day.

On the final pathology report, we found a $12 / 10 \mathrm{~cm}$ polypoid white greyish mass fixed to the posterior wall of the vagina with multiple foci of necrosis and hemorrhage. In addition, $1.5 \mathrm{~cm}$ ulceration in the anterior wall was noted.

On microscope, the tumoral proliferation was represented by multiple areas of spindle-shaped cells with eosionphilic cytoplasm. The nuclei were atypical sometimes giant with multiple abnormal mitosis (mitotic index corresponding to a grade III of Mac Govern). No lymph node involvement was found on histology. The tumor staged T1N0M0 according to the American joint committee.

Because of the tumor size, an adjuvant external radiotherapy at the dose of $50 \mathrm{~Gy}$ was delivered to the pelvis and inguinal areas. Twenty six months later, the patient was well with no sign of local or distant recurrence.

\section{Discussion}

The primary vaginal melanoma is rare. Less than 350 cases are described in the literature $[4,6]$. PARONA describe the first case in 1897. NYGOGOSSIAN et al demonstrate the presence of melanocytes in the basal part of the vaginal epithelium in the $3 \%$ of healthy women and this is the site that vaginal melanoma is thought to arise $[7,8]$.

It was reported that vaginal melanoma occurs in menopausal women in the $5^{\text {th }}$ and $6^{\text {th }}$ decades, while our patients were in their seventies [4].

The most common presenting symptoms are provoked or spontaneous vaginal bleeding, fetid vaginal discharge or a palpable mass [9]. In our cases, the symptoms were metrorrhagia and vaginal mass.

On physical examination, melanoma is reported as crumbly ulcerated nodular mass bleeding easily in contact, with a mean tumor size varying from 2 to $4 \mathrm{~cm}$, our mean tumor size were $7 \mathrm{~cm}$. The primary malignant melanoma of the vagina is reported to be located in $42.7 \%$ in lower third and in $38 \%$ in anterior wall of the vagina [9], in our cases the tumor were located in the lower third in one case and in the upper two thirds in the other but in the posterior wall of the vagina.

The differential diagnoses in surgical pathology are represented by the adenocarcinoma, small cell carcinoma and sarcomas, where the immunohistochemistry takes a place in the histological diagnosis of malignant melanoma using monoclonal antibodies S100 that represents the most sensitive biological marker of well differentiated melanocytes. The HMB-45 is more specific than the S100 antibody but less sensitive [2]. In our cases, the histological diagnosis was done using HMB45 and S100 antibodies.

There is no standard approach in the treatment of primary malignant melanoma of the vagina, because of the very low incidence of the unusual localization. The surgery remains the corner stone of the treatment but the extent of the resection ranges from conservative surgery such as wide excision of the lesion or total colpectomy to radical pelvic exenteration with en bloc removal of involved pelvic organs associated to inguinal and iliac lymphadenectomy [4, 7].

Some authors find that vaginal melanoma is an aggressive tumor with a high rate of local recurrence ranging from $22 \%$ to $80 \%$ and recommend radical surgery with lymphadenectomy [4]. Geisler et al. advised primary pelvic exenteration for vaginal melanoma over $3 \mathrm{~mm}$ of invasion and showed that 50\% 5-year survival rate might be obtained if the pelvic nodes were free of metastases [10]. Chung and Ariel, with separate series of 19 and 48 patients, respectively, defend the opinion that the appropriate management of this disease requires radical extirpation of the vagina supplemented by dissection of the regional lymphatics [11, 12]. Frumovitz and al performs surgery wide local excision or radical surgery in $76 \%$ of cases and exenteration in $14 \%$ cases. [4] 
On the other hand, some authors defend the conservative management of malignant vaginal melanoma giving as argument the presence of distant micro metastases at the diagnosis not detected at the initial work up, making an extended mutilating surgery ineffective [7, 13].

Our patients were treated with radical surgery associated to bilateral lymphadenectomy.

A few articles are dealing with sentinel lymph node mapping in malignant vaginal melanoma, only six cases are reported in the literature, to our knowledge [14]. An inguinal sentinel lymph node is detected in four patients with melanoma of the lower third of the vagina with no involvement on histology study [7, 14]. The authors concluded that the techniques of sentinel node must be performed in selected cases with no palpable node. The radiotherapy, is used when surgery is contraindicated, or in neoadjuvant setting to reduce the tumor size allowing a conservative resection. In adjuvant setting, the radiotherapy is related to a better local control and survival even after wide excision [2].

Frumovitz and al reported a reduced local recurrence risk and increased survival from 16.1 months to 29.4 months, although the increase was not statistically significant, when radiotherapy is used after complete resection, while Samolis states that radiotherapy, chemotherapy or radical extirpation cannot increase the survival time even in metastatic disease $[4,15]$. Ohno suggests that carbon ion radiotherapy can be the only conservative management for malignant melanoma of the vagina showing no evidence of local recurrence [16]. We used adjuvant radiotherapy for the second patient because of the important tumor size with no sign of local or distant recurrence.

Chemotherapy and immunotherapy may lead to excellent results. In fact, Wang and al found that surgery followed by chemotherapy and immunotherapy is the best therapeutic approach [17]. In a randomized study, Kirkwood and al demonstrate that IFN alpha- $2 \mathrm{~b}$ prolongs the time of tumor recurrence and improves overallsurvival. The prognosis of vaginal melanoma is poor; survival at 5 years is $8.4 \%$. Reid and al found that tumor size is the only significant prognostic factor: an analysis of 115 patients with lesions less than $3 \mathrm{~cm}$ shows a better survival in comparison with patients having lesions greater than $3 \mathrm{~cm}(\mathrm{P}=0.024)$. In fact, tumor size influences the range of remission and not overall survival [9].

\section{Conclusion}

With the experience of these 2 cases, it can be concluded that though Radical surgery is the treatment of choice for malignant melanoma of vagina and adjuvant Radiotherapy may improve survival by preventing recurrence. The literature review also supports Radiotherapy can prolong survival according few authors. Chemotherapy and immunotherapy may be combined when feasible with expectation of prolonged survival.

\section{References}

[1]. Greggi, S., S. Losito, C. Pisano, S. Desicato, and C. Scaffa, Malignant melanoma of the vagina: report of two cases and review of the literature. Int Surg 2010; 95: 120-5.

[2]. Gupta, D., A. Malpica, M.T. Deavers, and E.G. Silva, Vaginal melanoma: a clinicopathologic and immunohistochemical study of 26 cases. Am J Surg Pathol 2002; 26: 1450-7.

[3]. Tcheung, W.J., M.A. Selim, J.E. Herndon, 2nd, A.P. Abernethy, and K.C. Nelson, Clinicopathologic study of 85 cases of melanoma of the female genitalia. J Am Acad Dermatol 2012; 67: 598-605.

[4]. Frumovitz, M., M. Etchepareborda, C.C. Sun, et al., Primary malignant melanoma of the vagina. Obstet Gynecol 2010; 116: 1358-65.

[5]. Gungor, T., S.O. Altinkaya, M. Ozat, H. Bayramoglu, and L. Mollamahmutoglu, Primary malignant melanoma of the female genital tract. Taiwan J Obstet Gynecol 2009; 48: 169-75.

[6]. Mihajlovic, M., S. Vlajkovic, P. Jovanovic, and V. Stefanovic, Primary mucosal melanomas: a comprehensive review. Int J Clin Exp Pathol 2012; 5: 739-53.

[7]. Gokaslan, H., A. Sismanoglu, T. Pekin, H. Kaya, and N. Ceyhan, Primary malignant melanoma of the vagina: a case report and review of the current treatment options. Eur J Obstet Gynecol Reprod Biol 2005; 121: 243-8.

[8]. Nigogosyan, G., S. Delapava, and J.W. Pickren, Melanoblasts in Vaginal Mucosa. Origin for Primary Malignant Melanoma. Cancer 1964; 17: 912-3.

[9]. Reid, G.C., R.W. Schmidt, J.A. Roberts, M.P. Hopkins, R.J. Barrett, and G.W. Morley, Primary melanoma of the vagina: a clinicopathologic analysis. Obstet Gynecol 1989; 74: 190-9.

[10]. Geisler, J.P., K.Y. Look, D.A. Moore, and G.P. Sutton, Pelvic exenteration for malignant melanomas of the vagina or urethra with over $3 \mathrm{~mm}$ of invasion. Gynecol Oncol 1995; 59: 338-41.

[11]. Chung, A.F., M.J. Casey, J.T. Flannery, J.M. Woodruff, and J.L. Lewis, Jr., Malignant melanoma of the vagina--report of 19 cases. Obstet Gynecol 1980; 55: 720-7.

[12]. Ariel, I.M., Malignant melanoma of the female genital system: a report of 48 patients and review of the literature. J Surg Oncol 1981; 16: $371-83$.

[13]. Li, Y., M. Li, and Q. Wu, [Clinical analysis of 25 cases of primary vaginal malignant melanoma]. Zhonghua Fu Chan Ke Za Zhi 1999; 34: 162-4.

[14]. Abramova, L., J. Parekh, W.P. Irvin, Jr., et al., Sentinel node biopsy in vulvar and vaginal melanoma: presentation of six cases and a literature review. Ann Surg Oncol 2002; 9: 840-6.

[15]. Samolis, S., P. Panagopoulos, N. Kanellopoulos, I. Papastefanou, S. Karadaglis, and M. Katsoulis, Primary melanoma of the vagina: a case report. Eur J Gynaecol Oncol 2010;31: 233-4.

[16]. Ohno, T., S. Kato, E. Sasaki, K. Mizutani, and H. Tsujii, Carbon ion radiotherapy for vaginal malignant melanoma: a case report. Int J Gynecol Cancer 2007; 17: 1163-6.

[17]. Wang, Y., W. Chang, and D. Pu, [Clinical analysis of 15 patients with primary malignant melanoma in the genital tract]. Zhonghua Fu Chan Ke Za Zhi 1997; 32: 226-8. 